\title{
First Korean Case of Partial D DBS-1
}

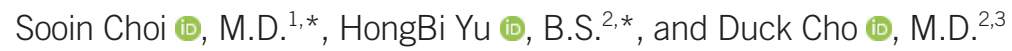 \\ ${ }^{1}$ Department of Laboratory Medicine, Soonchunhyang University Hospital Cheonan, Soonchunhyang University College of Medicine, Cheonan, Korea; \\ ${ }^{2}$ Department of Health Sciences and Technology, Samsung Advanced Institute for Health Sciences and Technology, Sungkyunkwan University, Seoul, Korea; \\ ${ }^{3}$ Department of Laboratory Medicine and Genetics, Samsung Medical Center, Sungkyunkwan University School of Medicine, Seoul, Korea
}

\section{Dear Editor,}

RHD and RHCE, encoding Rhesus proteins, are highly homologous genes located adjacently on the same chromosome (chromosome 1). Therefore, hybrid RHD genes, in which some portions are substituted with the RHCE sequence can change the extracellular loop of the RhD antigen, leading to variable reactivity to anti-D reagents [1].

Partial D phenotypes have historically been classified using epitope studies [2] and, recently, using genetic studies. DBS is a partial $\mathrm{D}$ phenotype characterized by c.[676G $>C]+[697 \mathrm{G}>\mathrm{C}]$ (NM_016124.4) and has been named based on its positive reactivity with the D monoclonal antibodies (MoAbs) BS228 and BS233 (Biotest, Dreieich, Germany) [3]. Three DBS subtypes have been reported till date: DBS-O [1], DBS-1 [3, 4], and DBS-2 [5]. Molecular studies have identified DBS-1 in an Arabian [3] and a Japanese family [4]; however, it is unknown whether DBS-1 correlates with the same hybrid gene of a single evolutionary origin in other, unrelated individuals from different ethnic groups. To the best of our knowledge, this is the first report of a DBS-1 case in a Korean family. This study was approved by the Institutional Review Board of Samsung Medical Center, Seoul, Korea (SMC-2019-11-160), and written informed consent was obtained from the proband and all family members.

The proband was a Korean woman with fibrocystic breast chan- ges, who was admitted to the Samsung Medical Center. D typing using anti-D Bioclone (MAD2 clone; Ortho Clinical Diagnostics, Raritan, NJ, USA) was negative. Weak D testing using antiD Bioclone and human IgG/IgM monoclonal anti-D (Millipore, Livingston, UK) yielded a result of grade 2+, while the result of partial D testing using D-screen (Diagast, Loos, France) was consistent with DBS-1. The phenotypes of the current case and previously reported DBS cases are summarized in Table 1.

The RhCE phenotype was ccEe (anti-C, -C, -E, and -e antibodies were obtained from Bioclone, Ortho Clinical Diagnostics, Buckinghamshire, UK), and RHD genotyping was carried out according to a previously described method [6]. Exon 5 was not amplified by the primer sets used in this study, but the region from exon 4 to exon 6 was amplified and the PCR product was sequenced using RHCE exon 5-specific primers; the Rhesus box was also PCR-amplified (Fig. 1A). The proband harbored a hybrid $R H D-c E(5)-D(D B S / d)$ allele involving the following amino acid changes: F223V, A226P, E233Q, V238M, V245L, G263R, and K267M. RHD genotypes of other family members are shown in Fig.1B. The breakpoints were confirmed between exon 4 and exon 6 by intron analysis $[3,4]$. The $5^{\prime}$ breakpoint region was the same as that reported by Omi, et al. [4], whereas the 3' breakpoint region was novel (Fig. 1C).

In various partial D phenotypes, such as DIIla, DVa, DVI, DAR,
Received: August 1, 2019

Revision received: August 18, 2019

Accepted: November 12, 2019

Corresponding author: Duck Cho, M.D.

Department of Laboratory Medicine and Genetics, Samsung Medical Center, Sungkyunkwan University School of Medicine, 81 Irwon-ro, Gangnam-gu, Seoul 06351, Korea

Tel: +82-2-3410-2403, Fax: +82-2-3410-2719, E-mail: duck.cho@skku.edu

\section{c) (i) (5)}

\section{(C) Korean Society for Laboratory Medicine}

This is an Open Access article distributed under the terms of the Creative Commons Attribution Non-Commercial License (https://creativecommons.org/licenses/by-nc/4.0) which permits unrestricted non-commercial use, distribution, and reproduction in any medium, provided the original work is properly cited.

\footnotetext{
*These authors equally contributed to this study.
} 


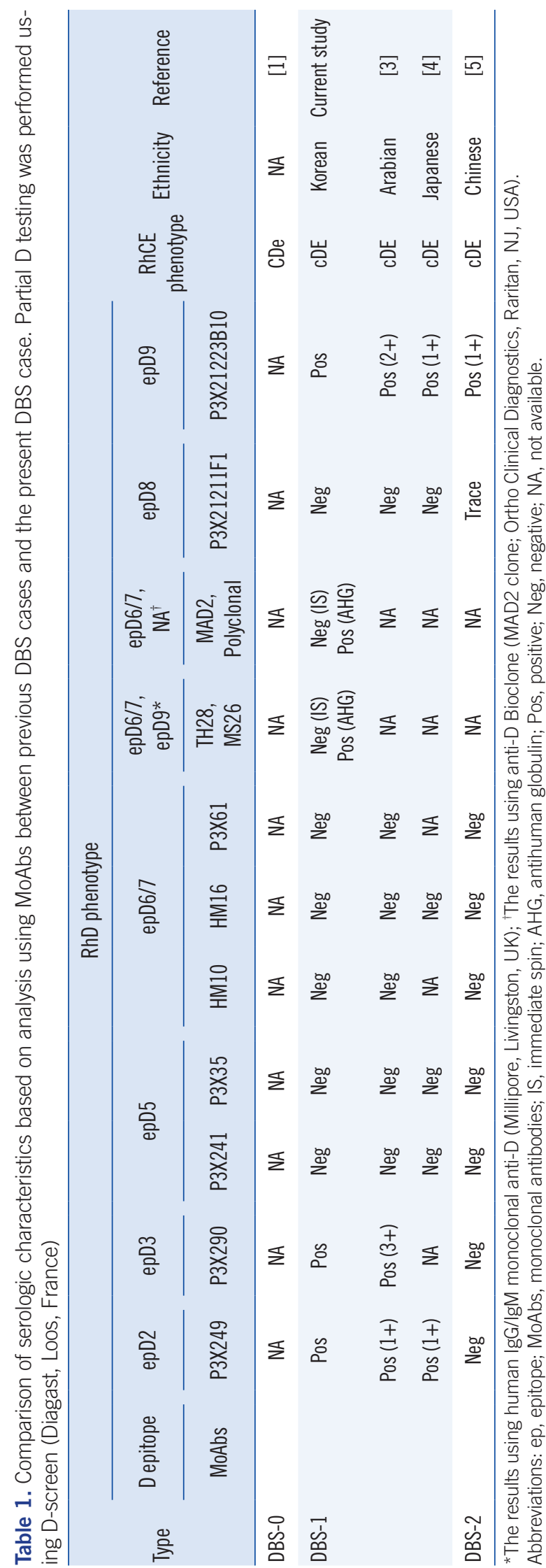

DFR, DBT, and DBS, RHD exon 5 is substituted with a part of RHCE exon 5 [7]. Exon 5 is predicted to encode the fourth extracellular loop of the D polypeptide. In DBS, the coexistence of two amino acid changes (A226P and E233Q) caused by c.676G >C and c.697G $>C$ point variants is required for the characteristic phenotype [4]. A226P is observed in the fourth loop of antigen $E$ [4] and is thought to have a considerable effect on D antigen density [1]. E233Q is also observed in the fourth loop as part of $\mathrm{D}^{\mathrm{w}}$ (RH23) [8]. DBS-1 and -2, which share F223V, A226P, and E233Q, exhibited different reactivity to the MoAbs P3X249, P3X290, and P3X21211F1 (DBS-1, +/+/- and DBS-2, -/-/trace). This difference might have been caused by the change in the extracellular amino acid, V238M, which is found in DBS-O and DBS-1, but not in DBS-2. Other amino acid changes observed in DBS-1, including V245L, G263R, and K267M, are located in the intracellular or transmembrane regions; however, they might affect the RhD phenotype.

The genetic basis of the RhD blood group differs across races and ethnicities. For example, the RhD-negative phenotype mainly results from $R H D$ deletion in Caucasians, whereas $R H D$ alterations, such as $R H D^{*} D-C E(4-7)-D$, are common in Africans [9]. Asians exhibit a high prevalence of C.1227G > A (NM_016124.4), known as "Asia type DEL." These ethnicity-specific trends are used not only to diagnose RhD variants, but also for transfusion protocols [10]. The 3' breakpoint observed in our case differed from that found in a Japanese family [4], suggesting that the DBS-1 cases have different genetic origins.

In conclusion, we reported the first case of DBS-1 in a Korean family. To understand the RhD characteristics specific to Korean ethnicity, further evaluation of RhD variants is required.

\section{ACKNOWLEDGEMENTS}

The authors wish to acknowledge the technical support of $\mathrm{Ji}$ Young Seo, M.T.

\section{AUTHOR CONTRIBUTIONS}

DC conceived the study; SC and HBY contributed to the interpretation of the results; all authors contributed to writing the manuscript.

\section{CONFLICTS OF INTEREST}

The authors declare no conflicts of interest. 
A

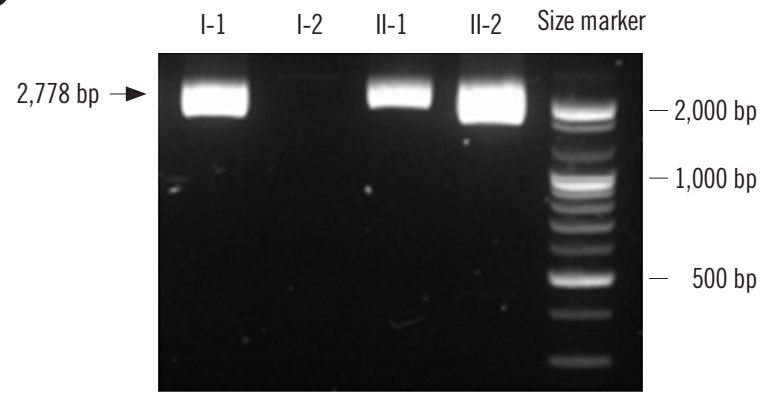

B

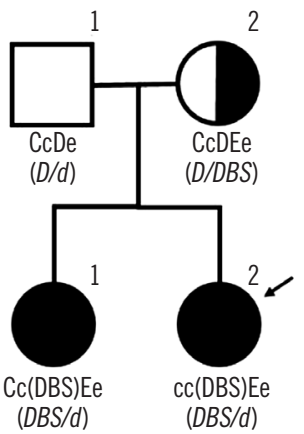

C

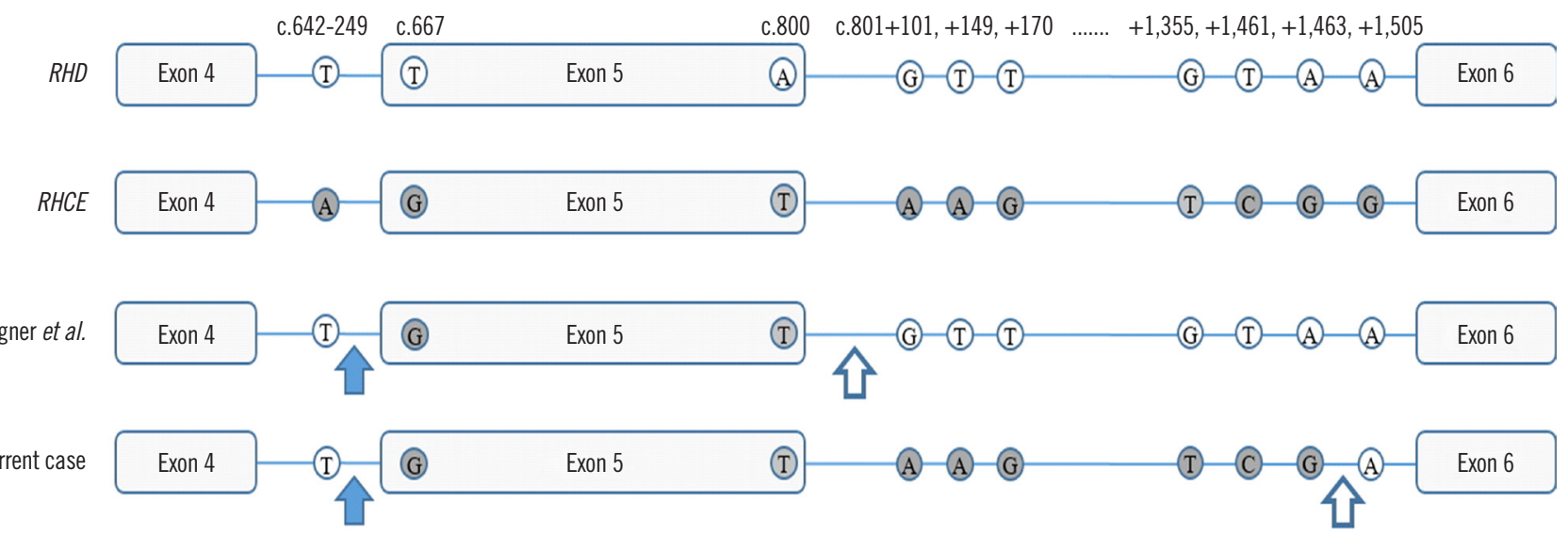

Fig. 1. Results of the genetic analysis of the proband and her family members. (A) Long-range PCR with primers located in non-Rhesus box sequences. A 2,778-bp fragment was amplified by PCR, indicating the presence of a hybrid RHD gene (lanes I-1, II-1, and II-2). (B) Pedigree, Rh phenotypes, and RHD genotypes of the Korean DBS-1 family. The genotypes and phenotypes of the DBS-1 family were determined using combined data from sequencing analysis, hybrid Rhesus box PCR, and serological analysis. Black circles indicate the DBS1 phenotype. The proband is indicated by a black arrow. Total RHD deletion is denoted as " $d$ " in the genotype. (C) Part of the RHD nucleotide sequence in DBS reported by Wagner, et al. [3] and this case. In both cases, the 5' breakpoint region was located between the RHDspecific c.642-249T and the first RHCE-specific nucleotide, c.667G (blue arrow). The 3' breakpoint region, located between the last RHCEspecific nucleotide and the first RHD-specific nucleotide of intron 5, differed for each case; it was located between c.800 and c.801+101 in the case reported by Wagner, et al. [3] and between c.801+1463 and c.801+1505 in the current case (white arrow).

\section{RESEARCH FUNDING}

None declared.

\section{ORCID}

$\begin{array}{ll}\text { Sooin Choi } & \text { https://orcid.org/0000-0003-4746-4809 } \\ \text { HongBi Yu } & \text { https://orcid.org/0000-0002-2401-5958 } \\ \text { Duck Cho } & \text { https://orcid.org/0000-0001-6861-3282 }\end{array}$

\section{REFERENCES}

1. Avent ND, Finning KM, Liu W, Scott ML. Molecular biology of partial D phenotypes. Transfus Clin Biol 1996;3:511-6.

2. Flegel WA, Von Zabern I, Doescher A, Wagner FF, Vytisková J, Písačka M. DCS-1, DCS-2, and DFV share amino acid substitutions at the extracel-
Iular RhD protein vestibule. Transfusion 2008:48:25-33.

3. Wagner FF, Ernst M, Sonneborn HH, Flegel WA. A DV-like phenotype is obliterated by A226P in the partial D DBS. Transfusion 2001;41:1052-8.

4. Omi T, Takahashi J, Seno T, Tanaka M, Hirayama F, Matsuo M, et al. Isolation, characterization, and family study of DTI, a novel partial D phenotype affecting the fourth external loop of D polypeptides. Transfusion 2002;42:481-9

5. Ye L, Wang P, Gao H, Zhang J, Wang C, Li Q, et al. Partial D phenotypes and genotypes in the Chinese population. Transfusion 2012;52:241-6.

6. Fasano RM, Monaco A, Meier ER, Pary P, Lee-Stroka AH, Otridge J, et al. $\mathrm{RH}$ genotyping in a sickle cell disease patient contributing to hematopoietic stem cell transplantation donor selection and management. Blood 2010;116:2836-8.

7. Wagner F. The Human RhesusBase, version 2.4. http://www.rhesusbase.info (Updated on Dec 2018)

8. Omi T, Okuda H, Iwamoto S, Kajii E, Takahashi J, Tanaka M, et al. Detection of Rh23 in the partial D phenotype associated with the DVa category. Transfusion 2000;40:256-7.

9. Wheeler MM, Lannert KW, Huston H, Fletcher SN, Harris S, Teramura G, 
et al. Genomic characterization of the RH locus detects complex and novel structural variation in multi-ethnic cohorts. Genet Med 2019;21: 477.

10. Choi S, Chun S, Seo JY, Yang JH, Cho D. Planned transfusion of D-pos- itive blood components in an Asia type DEL patient: proposed modification of the Korean National Guidelines for Blood Transfusion. Ann Lab Med 2019;39:102-4. 